\title{
Continuum discretization methods in a composite particle scattering off a nucleus: Benchmark calculations
}

\author{
O. A. Rubtsova* and V. I. Kukulin ${ }^{\dagger}$ \\ Institute of Nuclear Physics, Moscow State University, RU-119991 Moscow, Russia \\ A.M. Moro \\ Departamento de FAMN, Universidad de Sevilla, Apartado 1065, E-41080 Sevilla, Spain
}

(Received 13 June 2008; published 9 September 2008)

\begin{abstract}
The direct comparison of two different continuum discretization methods toward the solution of a composite particle scattering off a nucleus is presented. The first approach-the continuum-discretized coupled-channel method-is based on the differential equation formalism, while the second one- the wave-packet continuum discretization method - uses the integral equation formulation for the composite-particle scattering problem. As benchmark calculations, we have chosen the deuteron off ${ }^{58} \mathrm{Ni}$ target scattering (as a realistic illustrative example) at three different incident energies: high, middle, and low. Clear nonvanishing effects of closed inelastic channels at small and intermediate energies are established. The elastic cross sections found in both approaches are very close to each other for all three considered energies.
\end{abstract}

DOI: 10.1103/PhysRevC.78.034603

PACS number(s): 25.10.+s, 25.45.De, 03.65.Nk, 21.45.-v

\section{INTRODUCTION}

Historically, the main progress in studying elastic and inelastic (breakup) composite projectile scattering off a heavy target was made within the continuum-discretized coupledchannel (CDCC) method [1-17] in the framework of the Schrödinger coupled-channel scheme. In the CDCC approach, the total three-body scattering wave function (initial versions of the method assumed the projectile to be a two-fragment nucleus) is expanded in a complete basis of the fragment relative motion of the projectile, and the unknown three-dimensional elastic and breakup channel wave functions of the projectile c.m. motion are obtained by solving a set of coupled-channel equations. This approach has been successfully applied in the field of nuclear reactions to find the scattering amplitudes of many particular processes for various types of projectiles such as deuterons, ${ }^{6} \mathrm{Li},{ }^{6} \mathrm{He},{ }^{7} \mathrm{Li},{ }^{12} \mathrm{C}$, etc. However, a strong discussion about the validity of the method arose [16-18] because of some unclear issues related to the treatment of the total wave function breakup components in the asymptotic region. So, to check the accuracy and reliability of the results attained within this approach, the convergence of the elastic and breakup amplitudes (with variation of the momentum bin widths and their number) was studied in detail for various approximation schemes [9]. That study demonstrated clearly that the approach leads to convergent results for any reasonable choice of the discretization scheme. In particular, it was shown in Ref. [9] (for the test case of deuteron scattering off a ${ }^{58} \mathrm{Ni}$ target) that the employment of a finite number of exact two-body scattering wave functions as a basis for the internal projectile motion with energies fixed at midpoints of the bins (the midpoint method) leads unexpectedly in general to slower

\footnotetext{
*rubtsova-olga@yandex.ru

${ }^{\dagger}$ kukulin@nucl-th.sinp.msu.ru

${ }^{\ddagger}$ moro@us.es
}

convergence for the elastic and breakup amplitudes than the usage of the two-body wave functions averaged inside the energy bins (the average method). This result seems rather unnatural because the use of averaged wave functions (i.e., wave packets) as a basis for the internal projectile motion leads to a wrong asymptotic behavior of the coupling potentials and scattering wave functions in breakup channels, while the employment of the exact wave functions of the projectile internal sub-Hamiltonian seems at first glance to be more adequate.

Thus, the energy averaging method for the projectile internal continuum wave functions seems to assist in getting faster convergence in the CDCC calculations. The averaging leads to normalized (i.e., of $L^{2}$ type) wave functions $\phi_{i}(\mathbf{r})$, depending on the relative coordinate between the two fragments. At the same time, the motion along the second, projectile centerof-mass coordinate $\mathbf{R}$ is considered in the CDCC approach without any averaging on energy. So, the three-body wave function for the $i$ th excited channel has an outgoing asymptotic behavior, i.e.,

$$
\Psi(\mathbf{r}, \mathbf{R}) \underset{\substack{R \rightarrow \infty \\ r \ll R}}{\longrightarrow} \phi_{i}(\mathbf{r}) \frac{\exp \left(\mathrm{i} K_{i} R\right)}{R} .
$$

Therefore above the three-body breakup threshold, the total wave function $\Psi(\mathbf{r}, \mathbf{R})$ vanishes at $r \rightarrow \infty$ (similar to the bound-state function), while the dependence of the total scattering wave function upon the projectile center-of-mass coordinate has the pure outgoing wave asymptotic behavior. Such an asymmetry for the motions along the two coordinates at energies above the breakup threshold appears to be not fully justified [16-18], because in this energy region the proper asymptotic behavior over all spatial variables might be important.

However, for the scattering of a weakly bound projectile (e.g., deuteron or ${ }^{7} \mathrm{Li}$ ) at relatively high incident energies ( $E \gtrsim 40 \mathrm{MeV}$ ), the motion along the projectile c.m. coordinate 
$\mathbf{R}$ proceeds much faster than the motion along the relative coordinate $\mathbf{r}$ (if the projectile c.m. energy is much larger than its binding energy). In this case, the breakup of the projectile takes place mostly outside the nuclear field of the target. In other words, the projectile is first excited into its internal-continuum states, and then (already outside the reaction region) the excited projectile breaks up into its fragments. Within this picture, the breakup of the projectile in the target field can be treated as a particular case of inelastic scattering into the projectile continuum states. Then a proper averaging over energy bins for the fragment motion in the discretized continuum gives just a discrete representation of an exact spectral density function of this subsystem. As a result, this discretized density function enters all the elastic and breakup amplitudes. Thus, the applicability condition for the treatment of the above processes within the CDCC method should be $E \gg E_{\text {binding }}$. However, when the projectile kinetic energy $E$ is comparable to or even a bit larger than its internal binding energy $E_{\text {binding }}$, the other asymptotic channels should be considered, and thus the coupled-channel Schrödinger equation formalism should be replaced by the respective Faddeev approach.

Thus, within its scope of validity, the CDCC approach can be considered as a rather useful and practical working method to handle the realistic problems of composite projectile scattering off a target. However it would be very important to test carefully the accuracy and predictive power of the CDCC results in some fully independent way based on a different approach. The present paper is devoted just to a careful comparison between the CDCC and one alternative method for a typical problem in the field.

The alternative approach which we have chosen for our study is a new integral equation approach based on a total continuum discretization [19-23] which was developed by two of the present authors some time ago. The approach is based on the wave-packet continuum discretization (WPCD) technique, which allows one to convert the three-body integral equations into the respective matrix equation with the matrix elements easily calculable in an analytical way. In the WPCD method, the motion along both radial coordinates $\mathbf{r}$ and $\mathbf{R}$ is considered symmetrically, i.e., the respective averaged wave functions (stationary wave packets) vanishing at infinity along both coordinates are used. Such a fully discretized integral approach can be used because in the expression for the elastic scattering amplitude, the total three-body resolvent operator $G(E)$ is bracketed from the left and from the right by fast decaying factors over all the spatial coordinates:

$A_{\mathrm{el}}(E)=\left\langle\phi_{0}, \psi_{C}^{(-)}\left(E-\epsilon_{0}\right)|\bar{V}+\bar{V} G(E) \bar{V}| \phi_{0}, \psi_{C}^{(+)}\left(E-\epsilon_{0}\right)\right\rangle$,

where $\left|\phi_{0}, \psi_{C}^{( \pm)}\left(E-\epsilon_{0}\right)\right\rangle$ is the initial (final) projectile threebody wave function, so that the "external" interaction, $\bar{V}$, decaying along the c.m. coordinate $R$ and the projectile bound-state wave functions $\phi_{0}(\mathbf{r})$ decaying along the internal coordinate $r$ cuts effectively the asymptotic parts of the total resolvent $G(E)$. Thus, instead of the exact three-body resolvent operator $G(E)$, its respective wave-packet finite-dimensional approximation $\hat{G}(E)$ can be favorably used $[21,23]$. Therefore, in this integral wave-packet approach, we do not need any asymptotic parts of scattering wave functions. All that is required is just the inner parts of the wave functions but with the proper normalization, which can be well approximated via the stationary wave packets in the WPCD approach. It should be stressed that the effective cutoff of peripheral three-body resolvent parts does not depend on the total energy. Hence, if neglecting the stripping channels (similar to the conventional CDCC method) and treating the elastic and breakup channels only, the wave-packet method looks fully justified at low and high energies as well.

Such a "totally discretized" integral technique has several evident advantages over other formulations of the same problem. In addition to offering a convenient matrix scheme for calculating both the elastic and breakup amplitudes, it also allows one to construct in an explicit form an optical (energydependent and nonlocal) effective potential for the projectiletarget interaction which properly accounts for the intermediate inelastic processes $[21,23]$. Another distinctive feature of the integral approach is the proper and straightforward inclusion of closed channels, which are treated in this approach on the same footing as the open channels (see especially Sec. III).

So, to make the comparison between the two approaches maximally broad, we considered the deuteron elastic scattering by ${ }^{58} \mathrm{Ni}$ at three different incident energies: high, intermediate, and low, where closed-channel contributions are expected to be quite different. This reaction has been also used to compare the CDCC and Faddeev approaches in a recent work [24], showing very good agreement between both methods. It will be vividly demonstrated that the contribution of the closed channels, which are commonly neglected in CDCC calculations $[1-9,12]$, increases essentially when the projectile energy gets low. In principle, closed channels can be incorporated within the CDCC scheme; however, in this case the solution of the coupled equations becomes numerically unstable. In this situation, it is more convenient to solve the problem using the R-matrix method [25]. This is an equivalent way of solving the coupled equations, but with the advantage of being more stable numerically than the traditional radial stepping methods. However, this reformulation demands more computational resources than the conventional coupled-channel scheme [11]. Thus, we made a comprehensive comparison for both open and closed channel contributions between the R-matrix CDCC and WPCD approaches at different energies.

The paper is organized as follows. In Sec. II, a brief description of the CDCC and WPCD approaches is presented. Section III contains numerical results obtained with these two approaches, and the discussion is placed in Sec. IV. Finally, the summary of the paper is given in Sec. V.

\section{BRIEF DESCRIPTION OF THE CDCC AND WPCD APPROACHES}

The Hamiltonian describing the scattering of the twofragment composite particle $\{b c\}$ off the target nucleus $A$ is taken in the form

$$
\begin{aligned}
H= & h_{b c}(\mathbf{r})+h_{C}(\mathbf{R})+V_{b-A}\left(E_{\mathrm{lab}} / 2\right)+V_{c-A}\left(E_{\mathrm{lab}} / 2\right) \\
& +\Delta V_{C},
\end{aligned}
$$


where $h_{b c}$ is the sub-Hamiltonian of the internal $b-c$ motion (acting on the fragment relative coordinate $\mathbf{r}$ ), $h_{C}$ is the sub-Hamiltonian of the projectile center-of-mass asymptotic motion including the long-range point-like Coulomb interaction (acting along the c.m. coordinate $\mathbf{R}$ ), and $V_{c-A}$ and $V_{b-A}$ are energy-dependent optical potentials for the fragment-target system taken at half the incident particle energy $E_{\text {lab }}$ (for the nearly equal mass fragments). Finally, $\Delta V_{C}$ is the additional short-range Coulomb interaction (acting along the c.m. coordinate) which is caused by the finite charge radius of the target nucleus (due to charge distribution in the target nucleus). To obtain the elastic scattering and breakup amplitudes for the above scattering problem with the three-body Hamiltonian (1), one has to solve either the three-body Schrödinger equation with proper asymptotic boundary conditions or the system of the respective three-body Lippmann-Schwinger equations.

\section{A. Continuum discretized coupled-channel method (a differential equation formulation).}

In the CDCC approach, the continuous spectrum of the $h_{b c}$ sub-Hamiltonian is discretized by dividing the continuous momentum distribution into a finite number of nonoverlapping bins $\left[k_{i-1}, k_{i}\right]_{i=1}^{N}$ with corresponding averaged (within each bin) continuum wave functions $\left\{\left|\phi_{i}\right\rangle\right\}_{i=1}^{N}$ and respective averaged " channel eigenenergy" values $\epsilon_{i}^{*} \equiv\left\langle\phi_{i}\left|h_{b c}\right| \phi_{i}\right\rangle$. To simplify the notation, we omit here the angular variables, but they are assumed to be included. Then, the total three-body wave function of the Hamiltonian $H$ is expanded over the set of averaged wave functions describing the projectile internal spectrum (including the projectile bound state $\left|\phi_{0}\right\rangle$ with the binding energy $\left.\epsilon_{0}^{*}\right)$ :

$$
|\Psi(E)\rangle=\sum_{i=0}^{N}\left|\phi_{i}, \chi_{i}\right\rangle
$$

where $\left\langle\mathbf{R} \mid \chi_{i}\right\rangle \equiv \chi_{i}(\mathbf{R})$ are the channel wave functions that define the elastic $(i=0)$ and breakup $(i \neq 0)$ amplitudes. The bin wave functions $\left\{\left|\phi_{i}\right\rangle\right\}_{i=1}^{N}$ are typically constructed by averaging the true continuum states, $\phi(k)$ within the bin interval:

$$
\left|\phi_{i}\right\rangle=\frac{1}{\sqrt{\Delta_{i}}} \int_{k_{i-1}}^{k_{i}} f(k)|\phi(k)\rangle d k, \quad i=1, \ldots, N,
$$

where $\Delta_{i}$ is a normalization constant and $f(k)$ is a weight function. For a nonresonant continuum, the common choice is $f(k) \equiv 1$, in which case $\Delta_{i}=k_{i}-k_{i-1}$. Applying the expansion (2) to the initial Schrödinger equation for the Hamiltonian (1), one gets a system of coupled differential equations for the unknown functions $\chi_{i}(\mathbf{R})$ :

$$
\begin{aligned}
& {\left[h_{C}(\mathbf{R})+\Delta V_{C}(\mathbf{R})+V_{i i}(\mathbf{R})-\left(E-\epsilon_{i}\right)\right] \chi_{i}(\mathbf{R})} \\
& =\sum_{\substack{i=0 \\
i \neq i}}^{N} V_{i i}, \chi_{i \prime}(\mathbf{R}),
\end{aligned}
$$

where $V_{i i}(\mathbf{R}) \equiv\left\langle\phi_{i}\left|V_{b-A}+V_{c-A}\right| \phi_{i \prime}\right\rangle^{1}$ are coupling potentials. This system is solved with the following boundary conditions [9]:

$$
\chi_{i}(R) \sim I\left(K_{i}, R\right) \delta_{i 0}-\sqrt{\frac{K_{i}}{K_{0}}} S_{i, 0} O\left(K_{i}, R\right),
$$

where $I$ and $O$ are the Coulomb incoming and outgoing waves, $K_{i}$ is the c.m. wave number, which is related to the channel eigenenergy $\epsilon_{i}^{*}$ by energy conservation, i.e., $E=\epsilon_{i}^{*}+\frac{\hbar^{2} K_{i}^{2}}{2 M}$ (here $M$ is the deuteron-target reduced mass) and $S_{i, 0}$ are $S$-matrix elements for the elastic $(i=0)$ and inelastic $(i \neq 0)$ scattering. ${ }^{2}$

\section{B. Wave-packet continuum discretization method}

In the WPCD approach, one applies an analogous discretization of the continuous spectrum of the $h_{b c}$ subHamiltonian but making use of energy bins: $\left[\epsilon_{i-1}, \epsilon_{i}\right]_{i=1}^{N}{ }^{3}$. In a similar way, one defines a set of stationary wave packets (WPs) as integrals of the exact scattering wave functions $|\phi(E)\rangle$ of the $h_{b c}$ sub-Hamiltonian over the respective energy bins:

$$
\left|Z_{i}\right\rangle=\frac{1}{\sqrt{D}_{i}} \int_{\epsilon_{i-1}}^{\epsilon_{i}}|\phi(E)\rangle d E, \quad i=1, \ldots, N
$$

where $D_{i} \equiv \epsilon_{i}-\epsilon_{i-1}$ are the bin widths, and the corresponding channel eigenenergies are just the bin midpoints: $\epsilon_{i}^{*}=\frac{1}{2}\left(\epsilon_{i-1}+\epsilon_{i}\right)$. The set of these WP states should be supplemented with the bound state $\left|Z_{0}\right\rangle \equiv\left|\phi_{0}\right\rangle$. The main difference in this discretization procedure with respect to the conventional CDCC method is that we use here a full set of WP states which includes both open and closed channels.

Besides that, we build a partition of the continuous energy spectrum of the Coulomb sub-Hamiltonian $h_{C}$ acting along the projectile c.m. coordinate $\mathbf{R}$ and employ the corresponding set of Coulomb stationary wave packets (CPs) constructed by averaging the regular Coulomb continuum wave functions $\left|\psi^{C}(E)\right\rangle$ over energy bins $\left[\mathcal{E}_{j-1}, \mathcal{E}_{j}\right]_{j=1}^{M}$ :

$$
\left|X_{j}^{C}\right\rangle=\frac{1}{\sqrt{\Delta_{j}}} \int_{\mathcal{E}_{j-1}}^{\mathcal{E}_{j}}\left|\psi^{C}(E)\right\rangle d E,
$$

where $\Delta_{j} \equiv \mathcal{E}_{j}-\mathcal{E}_{j-1}$ are the bin widths. Finally, we construct the three-body wave-packet (TWP) basis set for the elastic channel asymptotic Hamiltonian,

$$
H_{b c}=h_{b c} \oplus h_{C},
$$

\footnotetext{
${ }^{1}$ The integral is taken over the relative $\mathbf{r}$ coordinate only.

${ }^{2}$ For closed channels $\left(\epsilon_{i}>E\right)$ the wave functions will contain an exponentially growing part and an exponentially decreasing part. The former tends to destroy the initially generated linear independence of the solution vectors. The longer the integration continues through a classically forbidden region, the stronger this tendency will be. Thus the closed channels are usually not taken into account in these calculations. To avoid this problem, the combination of the CDCC and R-matrix approaches can be used.

${ }^{3}$ It is possible to make just the same energy and momentum bins in the WPCD and CDCC approaches using the interrelation $\epsilon_{i}=\frac{\hbar^{2} k_{i}^{2}}{2 m}$, where $m$ is the reduced mass of the two projectile constituents.
} 
as the direct product of the states $\left|Z_{i}\right\rangle$ and $\left|X_{j}^{C}\right\rangle$ (including all the required partial wave couplings):

$$
\left|S_{i j}\right\rangle \equiv\left|Z_{i}, X_{j}\right\rangle, \quad i=0, \ldots, N ; \quad j=1, \ldots, M .
$$

Such a three-body packet basis (which is also of the $L^{2}$ type) is very convenient for the subsequent investigations. One of its main advantages is that the matrix of the channel resolvent $G_{b c} \equiv\left[E+\mathrm{i} 0-H_{b c}\right]^{-1}$ is diagonal in this basis. Moreover, the corresponding matrix elements of the three-body resolvent operator have an explicit analytical form [21,23], and they depend only on the spectrum discretization parameters (i.e., $\epsilon_{i}$ and $\mathcal{E}_{j}$ values).

Because the interactions between the projectile constituents and the target are given by complex optical-model potentials, the contribution of the intermediate rearrangement (viz. stripping) channels to the elastic scattering or breakup should be of minor importance. ${ }^{4}$ Therefore, to treat the projectile elastic scattering in the leading order, one needs to take into account only those intermediate breakup states whose asymptotic behavior are defined by the three-body channel Hamiltonian $H_{b c}{ }^{5}$ So, the total three-body scattering wave function $|\Psi(E)\rangle$ in this case can be found from a single Lipmann-Schwinger (LS) equation:

$$
|\Psi(E)\rangle=\left|\Psi_{d}(E)\right\rangle+G_{b c}(E) \bar{V}_{b c}|\Psi(E)\rangle,
$$

where $\bar{V}_{b c} \equiv V_{b-A}+V_{c-A}+\Delta V_{C}$ is the "external" shortrange interaction, and $\left|\Psi_{d}(E)\right\rangle \equiv\left|Z_{0}, \psi^{C}\left(E-\epsilon_{0}^{*}\right)\right\rangle$ is the outgoing wave function for the projectile in its ground state. The long-range Coulomb part for the point-like Coulomb interaction between the projectile and target is included in the $G_{b c}$ operator. Next, we make the projection of Eq. (10) onto the wave-packet subspace and expand the total wave function in the three-body wave-packet basis for the Hamiltonian $H_{b c}$ :

$$
|\Psi(E)\rangle \approx|\hat{\Psi}(E)\rangle=\sum_{i j} C_{i j}\left|S_{i j}\right\rangle .
$$

Then Eq. (10) can be rewritten in the finite-dimensional form

$$
|\hat{\Psi}(E)\rangle=\left|S_{0 j_{0}}\right\rangle+\hat{G}_{b c}(E) \hat{V}|\hat{\Psi}(E)\rangle,
$$

where $\left|S_{0 j_{0}}\right\rangle \equiv\left|Z_{0}, X_{j_{0}}^{C}\right\rangle$ is the packet state corresponding to the outgoing wave function $\left|\Psi_{d}\right\rangle$ (the index $j_{0}$ is defined by the energy rule: $\left.E \in\left[\mathcal{E}_{j_{0}-1}+\epsilon_{0}^{*}, \mathcal{E}_{j_{0}}+\epsilon_{0}^{*}\right]\right)$. The interaction operator $\hat{V}$ is the wave-packet projection of the external interaction $\bar{V}_{b c}$, that is,

$$
\hat{V} \equiv \sum_{i j, i j^{\prime}{ }^{\prime}}\left|S_{i \prime j^{\prime}}\right\rangle\left\langle S_{i j}\right| V_{i j, i, j},
$$

whose matrix elements in the TWP basis can be interpreted as generalized coupling potentials

$$
V_{i j, i j^{\prime} j} \equiv\left\langle S_{i j}\left|V_{b-A}+V_{c-A}+\Delta V_{C}\right| S_{i \prime j^{\prime}}\right\rangle
$$

\footnotetext{
${ }^{4}$ Due to the imaginary potentials, there are no bound states in the rearrangement channels, and the kinetic energy of the projectile is assumed to be relatively high.

${ }^{5}$ This is the same assumption as in the CDCC approach.
}

and may be calculated either analytically or numerically. Thus, in this approach, solving the three-body LS equation is reduced to the solution of the simple matrix equation (12). ${ }^{6}$

The composite-particle elastic scattering amplitude can now be written formally as the diagonal (on-shell) matrix element

$$
A_{\mathrm{el}} \approx \frac{\left[\left(\mathbf{V}^{-1}-\mathbf{G}_{b c}\right)^{-1}\right]_{0 j_{0}, 0 j_{0}}}{\Delta_{j_{0}}},
$$

where matrices of the respective operators in the three-body WP basis are denoted by the bold letters, while $\Delta_{j_{0}}$ is the bin width of the $h_{C}$ continuum partition. Moreover, the breakup amplitude can be readily found as a nondiagonal (off-shell) matrix element of the same matrix $\left[\mathbf{V}^{-1}-\mathbf{G}_{b c}\right]^{-1}$ in the WP basis $[21,23]$. The above matrix is essentially a finite-dimensional analog of the exact transition operator matrix.

Here it is appropriate to summarize the main distinctive features that distinguish the wave-packet approach from the conventional CDCC method:

(i) The wave-packet basis for the projectile bound and continuum internal states is chosen to go up to high excitation energies, so that not only open-channel but also closed-channel contributions are fully included in the WP three-body calculations.

(ii) The internal and external motions of the projectile are considered in the WP technique on an equal footing, i.e., using the bases of wave packets averaged over the respective energy bins for the motions along both independent radial coordinates. So the total threebody resolvent in the transition matrix elements is cut effectively at large distances by the interaction potentials and also by the bound-state functions of the projectile in the initial and final states.

(iii) The above-mentioned cutoff of the total three-body resolvent is intimately related to the integral formulation of the WP approach, in which a proper asymptotic behavior of coupling potentials and an explicit matching procedure between inner and asymptotic wave functions are not required.

(iv) The kernel of the respective matrix equation in the WP approach includes rather smoothed (on energy) matrix elements due to the integration over energy bins for the motions along all active coordinates. This important feature of WP matrix kernels can readily be extended to the solution of the general Faddeev equations using the WP technique [21].

(v) The integral formulation of the three- and few-body scattering problem makes it possible to reformulate easily the initial problem in terms of the Feshbach projection operator technique [23]. This reformulation

\footnotetext{
${ }^{6}$ It should be stressed that the matrix equation (12) in our case is strongly distinguished from that which can be deduced from a direct matrix reduction of the three-body LS equation using some quadrature mesh-points, because all the matrix elements in our case have been averaged over energy bins corresponding to both active coordinates.
} 
allows one to reduce the elastic scattering of a composite projectile to a simple potential scattering by a nonlocal and energy-dependent potential and moreover to construct this potential in an explicit analytical form.

So, it would be very instructive to make a direct comparison between the WPCD and the conventional CDCC results for some standard test problem.

\section{COMPARISON BETWEEN THE CDCC AND WPCD: BENCHMARK CALCULATIONS AT DIFFERENT INCIDENT ENERGIES}

As a general numerical test of the two methods, we have chosen the popular case of the elastic scattering of the deuteron off a ${ }^{58} \mathrm{Ni}$ target at three different incident energies, namely, $E_{\text {lab }}=80,21.6$, and $12 \mathrm{MeV}$. These detailed calculations allow us to not only make a wide comparison between the two approaches but also to deduce from this comparison the role of the closed channels at different energies.

For the sake of simplicity, the neutron-proton $(n-p)$ interaction is assumed here to have the Gaussian form [4]

$$
\begin{aligned}
V(r) & =-V_{0} e^{-\beta r^{2}}, \quad V_{0}=66.99 \mathrm{MeV}, \\
\beta & =0.415 \mathrm{fm}^{-2} .
\end{aligned}
$$

Also, keeping in mind the comparison purposes between the two alternative methods, we restrict ourselves here to $s$ waves for the $n$ - $p$ relative motion. ${ }^{7}$

The nucleon-nucleus interactions are represented by local optical-model potentials of Woods-Saxon type. In particular, we employ here the potentials from the Koning-Delaroche global fits [26] evaluated at half the deuteron incident energy. The mass values $m_{n}=1.0087 \mathrm{amu}$ (for the neutron), $m_{p}=$ $1.0078 \mathrm{amu}$ (for the proton), and $m_{\mathrm{Ni}}=57.9353 \mathrm{amu}$ (for the target) are adopted here.

\section{A. Choices for parameter values of the momentum distribution in the CDCC method}

Following the standard procedure, in the CDCC calculations the continuum was truncated by defining a maximum excitation $E_{\max }$, and divided into $N$ energy bins, evenly spaced in the linear momentum. For each bin, a representative wave function was constructed according to Eq. (3) with $f(k)=1$. The number of bins $N$ was determined in order to achieve convergence of the elastic angular distribution. For the two lower energies, $E_{\text {lab }}=12 \mathrm{MeV}$ and $E_{\text {lab }}=21.6 \mathrm{MeV}$, the calculations included both open and closed channels. Thus, the continuum was represented by a set of $N_{\text {open }}$ bins up to the maximum available c.m. energy, and by $N_{\text {closed }}$ bins from this energy to $E_{\max }$, and hence $N=N_{\text {open }}+N_{\text {closed }}$.

\footnotetext{
${ }^{7}$ Higher $n$ - $p$ partial waves would be certainly important to comparing the theoretical results with the respective experimental data.
}

\section{B. Choices for parameter values of the wave-packet bases for the WPCD calculations}

To simplify the construction of the $n$ - $p$ relative motion WPs, $\left|Z_{i}\right\rangle$, and to get simultaneously the bound-state wave function, $\left|Z_{0}\right\rangle$, we apply here a single diagonalization procedure for the $h_{b c}$ sub-Hamiltonian on the simple Gaussian basis $\left|\psi_{n}\right\rangle_{n=1}^{N_{G}}$, i.e., we assume that the $n-p$ wave packets are expanded as follows:

$$
\left|Z_{i}\right\rangle \approx \sum_{n=1}^{N_{G}} A_{n}\left|\psi_{n}\right\rangle, \quad \psi_{n}(r)=B_{n} e^{-\alpha_{n} r^{2}},
$$

where $B_{n}$ are normalization factors. The scale parameters $\alpha_{n}$ are defined on the generalized Tchebyshev grid

$$
\alpha_{n}=\alpha_{0}\left[\tan \left(\frac{\pi(2 n-1)}{4 N_{G}}\right)\right]^{t}, \quad n=1, \ldots, N_{G},
$$

where $\alpha_{0}$ is the common scale parameter, and $t$ defines the distribution sparseness for the Gaussian "frequencies" $\alpha_{n}$. As $t$ becomes larger, the mesh points $\alpha_{n}$ are simultaneously extended to lower and higher values, so that longer range and shorter range Gaussian components appear in the basis. The energy distribution $\epsilon_{i}^{*}$ (and the corresponding wave numbers $k_{i}=\sqrt{2 m \epsilon_{i}^{*} / \hbar^{2}}$ ) obtained with such a basis turns out to be highly nonuniform (i.e., not equidistant), but it covers practically all the energy spectrum and provides well-converged results for the scattering amplitudes [19,20]. Figure 1 shows the energy and corresponding momentum distributions for the following parameter values chosen for the grid: $N_{G}=20, t=2$, and $\alpha_{0}=0.1 \mathrm{fm}^{-2}$. In practical calculations, we found that it is quite sufficient to include only the first $N$ basis functions with maximum bin energy $\epsilon_{N}$ less than some chosen maximal energy value $E_{\max }$ of the included closed channels. For example, it is clear from Fig. 1 that a basis containing the first $N=13$ functions is enough for $E_{\max }=100 \mathrm{MeV}$.

As for the WP basis to describe the $n$ - $p$ pair c.m. motion we employ (for all required partial waves $L$ ) a set of the exact

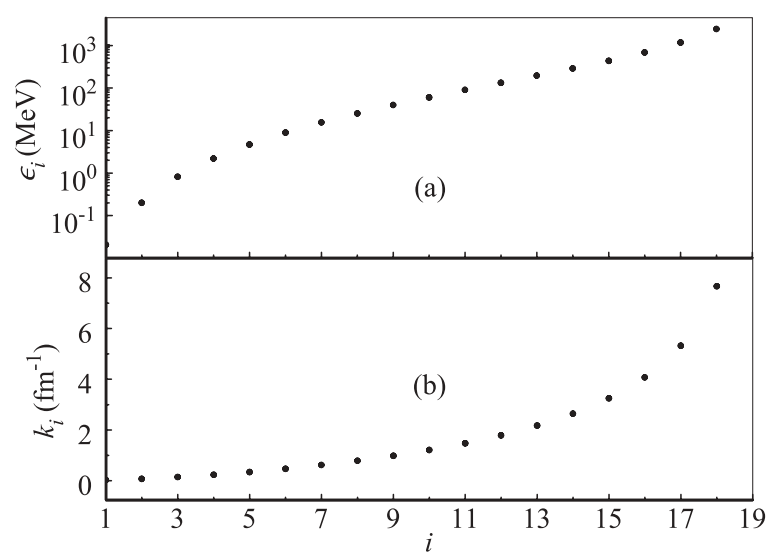

FIG. 1. Energy $\epsilon_{i}$ (a) and momentum $k_{i}$ (b) distributions for the $n-p$ continuum discretization constructed with a Gaussian basis on the generalized Tchebyshev grid (18). 
Coulomb packets of the dimension $M$ constructed also on the Tchebyshev grid with $t=1$, which corresponds to the energy distribution

$$
\mathcal{E}_{j}=E_{0} \tan \left(\frac{\pi(2 j-1)}{4 M}\right), \quad j=1, \ldots, M,
$$

where $\mathcal{E}_{j}$ are the end points of the energy bins $\left(\mathcal{E}_{0}=0\right)$, and $E_{0}$ is the incident energy of the projectile in the c.m. frame.

\section{Results of calculations}

In this section we will present the particular results for the differential elastic cross sections calculated at different incident energies.

\section{1. $E_{\text {lab }}=80 \mathrm{MeV}$}

At this relatively high energy, we found that the breakup effect contribution is rather small, producing just a minor correction to the folding model result (i.e., the calculation omitting continuum channels). Also it was shown previously [9] that only about half of the open channels (i.e., $E_{\max } \approx$ $40 \mathrm{MeV}$ ) play a significant role at this relatively high energy. So one can conclude that the effect of closed channels can be ignored here. Figure 2 compares the elastic differential cross sections obtained within the CDCC, the WPCD, and the folding model calculations. For these calculations, the WP bases with dimensions $N=13$ (for $n$ - $p$ relative motion) ${ }^{8}$ and $M=500$ (for deuteron c.m. motion) are used. The number of partial waves required for the deuteron-target relative motion is $L_{\max }=45$. For the CDCC calculations, the continuum was truncated at $E_{\max }=70 \mathrm{MeV}$ and divided into $N=20$ bins, uniformly distributed in the linear momentum $k$. The set of coupled equations were solved using the Numerov method

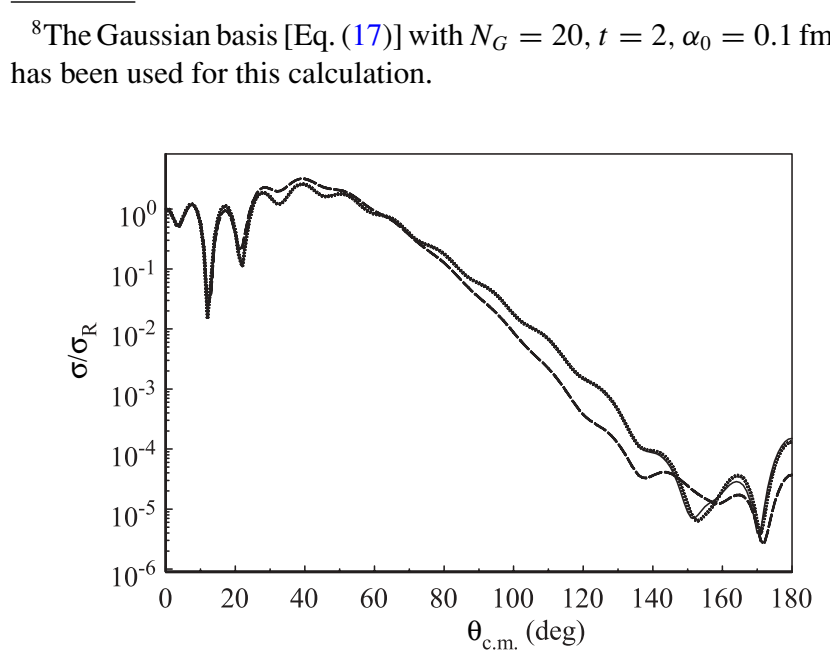

FIG. 2. Comparison of the differential elastic cross sections of the deuteron off ${ }^{58} \mathrm{Ni}$-target scattering (as a ratio to the Rutherford cross section $\sigma_{\mathrm{R}}$ ) at $E_{\text {lab }}=80 \mathrm{MeV}$ calculated with the CDCC (dotted line) and WPCD approaches (full line). The dashed curve corresponds to the single folding model calculation. and matched to their asymptotic solution at $20 \mathrm{fm}$. These calculations were performed with the code FRESCO [11].

As it follows from Fig. 2, the results of both discretization methods are in very good agreement for all angles. It should be emphasized that despite the highly nonhomogeneous bin distributions for the internal $n-p$ subsystem as well as for the deuteron-target sub-Hamiltonian, the WPCD calculations led to well-converged results. So, in practical calculations, it is not necessary to use only equidistant momentum bin distributions as is usually done $[4,7,9]$. Moreover, the application of some special energy distribution allows one to decrease significantly the number of packet basis functions required for convergence. ${ }^{9}$ Thus, to summarize, despite the quite different bin distributions considered in the CDCC and WPCD approaches and their rather different formalisms, both methods provide results that nicely agree with each other at $E_{\text {lab }}=80 \mathrm{MeV}$.

\section{2. $E_{\mathrm{lab}}=21.6 \mathrm{MeV}$. The effect of closed channels}

At this intermediate energy, the influence of closed channels (CC) on the elastic scattering amplitude becomes visible. Our calculations have shown that the main part of this effect is seen at backward angles. Though the $\mathrm{CC}$ effect has been commonly neglected in the conventional CDCC calculations $[1-9,12]$, it is worth studying carefully the convergence of the elastic cross section when the number of closed channels and the corresponding maximum energy $E_{\max }$ are increased.

To prepare these calculations in the WPCD scheme, we used the three-body WP sets of dimensions $N=15$ [constructed with the Gaussian basis (17) with $N_{G}=30, t=3$, and $\alpha_{0}=$ $0.1 \mathrm{fm}^{-1}$ ] and $M=150$. The number of partial waves required at this energy is $L_{\max }=25$. To incorporate the closed channels into the CDCC framework, a special combination of the coupled-channel scheme and the R-matrix approach has been used [25]. In contrast to the Numerov method, where the radial functions $\chi_{i}(\mathbf{R})$ are obtained by direct integration of the coupled differential equations (4), in the R-matrix method a basis set of energy eigenstates is first obtained by solving the diagonal parts of the coupled equations, with the basis functions all having fixed logarithmic derivatives at a given distance $R_{m}$. Next, the unknown functions $\chi_{i}(\mathbf{R})$ are expanded in this basis. Although this procedure is computationally more demanding, it leads to more stable results when closed channels are taken into account. To achieve convergence at this incident energy, we required $N_{\text {open }} \simeq 20$ and $N_{\text {closed }} \simeq$ 15 bins, for a maximum excitation energy $E_{\max } \sim 140 \mathrm{MeV}$. The R-matrix radius was fixed to $R_{m}=30 \mathrm{fm}$.

In Fig. 3, the comparison between the R-matrix CDCC and the WPCD elastic cross sections calculated at almost equal $E_{\max }$ values is presented. For $E_{\max }=20 \mathrm{MeV}$, there are only open channels, and the conventional Numerov procedure has been employed to solve the CDCC equations [see Fig. 3(a)].

\footnotetext{
${ }^{9}$ This feature is also useful in the presence of a resonance in the $b-c$ continuum. The general WPCD formalism remains the same for this case, but some detailed energy distribution is required (see Ref. [20] with the $\alpha-\alpha$ scattering problem).
} 


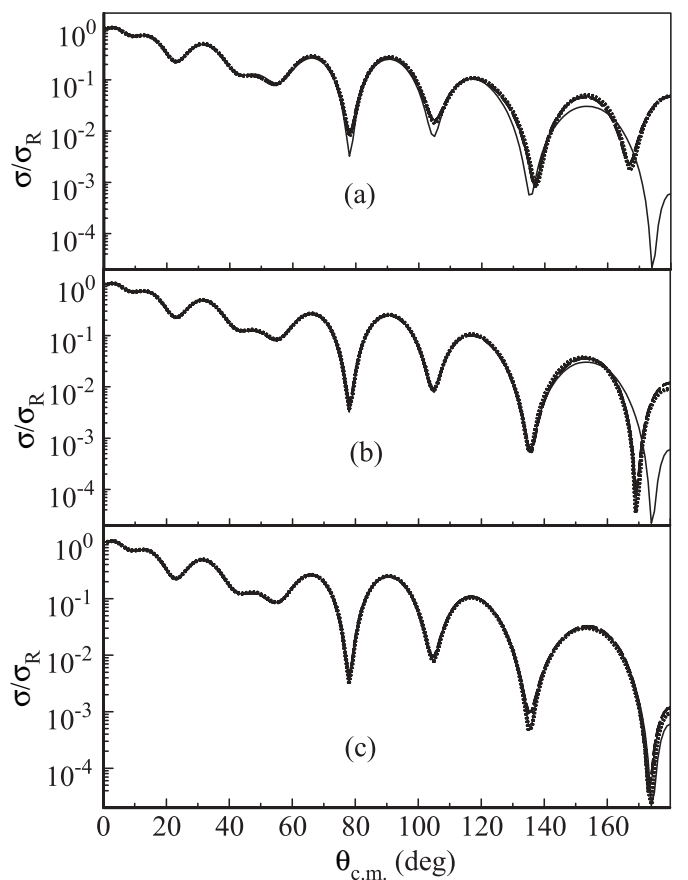

FIG. 3. Elastic $d+{ }^{58} \mathrm{Ni}$ cross section at $E_{\text {lab }}=21.6 \mathrm{MeV}$ calculated within the CDCC (dotted line) and WPCD (dashed line) approaches for different values of $E_{\max }$ : (a) $20 \mathrm{MeV}$, (b) $38 \mathrm{MeV}$, and (c) $80 \mathrm{MeV}$. The full lines correspond to the converged CDCC and WPCD results (with $E_{\max }=139 \mathrm{MeV}$ ), which are almost indistinguishable in these plots.

For the other values of $E_{\max }$ [Figs. 3(b) and 3(c)], the Rmatrix method has been employed, while the WPCD technique has not been changed. It is evident that the results for the differential cross sections found with the WPCD and R-matrix CDCC methods with similar $E_{\max }$ values are almost identical. So, it can be concluded that the details of the discretization distributions in both methods do not play an important role in the convergence, and the most significant factor here is just the $E_{\max }$ value.

The converged elastic cross sections (calculated with the highest value, $E_{\max }=139 \mathrm{MeV}$ ) are presented in Fig. 4. The curves corresponding to both discretization methods are hardly distinguishable. In this figure we have also included the calculation corresponding to the folding model calculation (dashed line). It is evident that breakup effects are large at this incident energy.

In Fig. 5, we plot the elastic $S$-matrix elements obtained with both discretization methods. It can be seen that they are in very good agreement for all partial waves.

From the present results it becomes clear that the CC effect must be taken into account at these intermediate energies. Furthermore, the required maximum energy of the CCs is rather high, viz. $E_{\max } \sim 100 \mathrm{MeV}$.$$
\text { 3. } E_{\mathrm{lab}}=12 \mathrm{MeV}
$$

We finally present in this section the calculations at the relatively low energy of $12 \mathrm{MeV}$. In this case, the dimensions of the WP sets $N=15$ (constructed from the Gaussian basis (17)

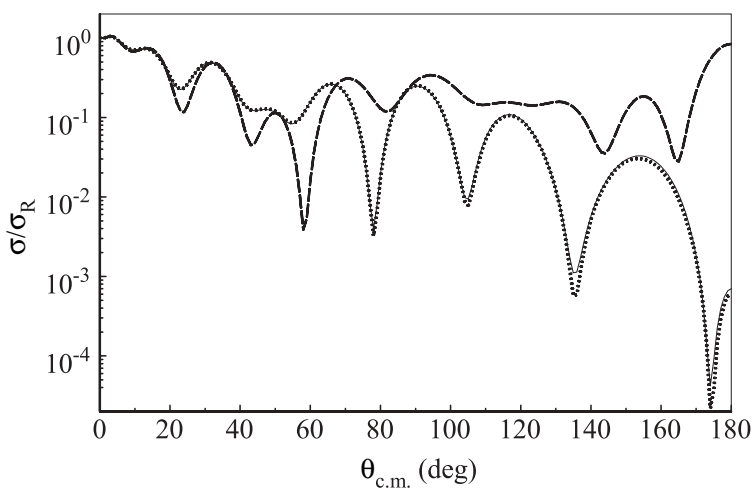

FIG. 4. Converged results (with respect to $E_{\max }$ ) for the differential elastic cross section (relative to Rutherford) for $d+{ }^{58} \mathrm{Ni}$ at $E_{\text {lab }}=21.6 \mathrm{MeV}$, calculated with the CDCC (dotted curve) and WPCD (solid curve) approaches. The dashed line corresponds to the folding calculation, in which continuum channels are not taken into account.

with $N_{G}=30, t=3$, and $\alpha_{0}=0.1 \mathrm{fm}^{-2}$ ) and $M=150$ are sufficient, and the maximum deuteron c.m. angular momentum value is only $L_{\max }=15$. For the CDCC calculations, we used $N_{\text {open }}=15$ and $N_{\text {closed }}=10$ (for $E_{\max } \sim 80 \mathrm{MeV}$ ). Again, the $\mathrm{R}$-matrix technique was used to solve the coupled equations, with a matching radius of $R_{m}=30 \mathrm{fm}$.

The elastic cross sections calculated with the WPCD and the R-matrix CDCC approaches for different $E_{\max }$ values are shown in Fig. 6. The comparison between the converged (with respect to $E_{\max }$ values) cross sections calculated with both methods along with the folding model results are presented in Fig. 7.

It is evident that the influence of the $\mathrm{CC}$ is the largest here, and this effect is seen not only at backward angles but also at intermediate angles $\left(\theta_{\text {c.m. }} \sim 90^{\circ}-100^{\circ}\right)$. The small discrepancies between the WPCD and R-matrix CDCC results observed at most backward angles might be ascribed to the different $n-p$ continuum discretization parameters, but we cannot rule out that they can be related to numerical inaccuracies of either of the two methods. In any case, this

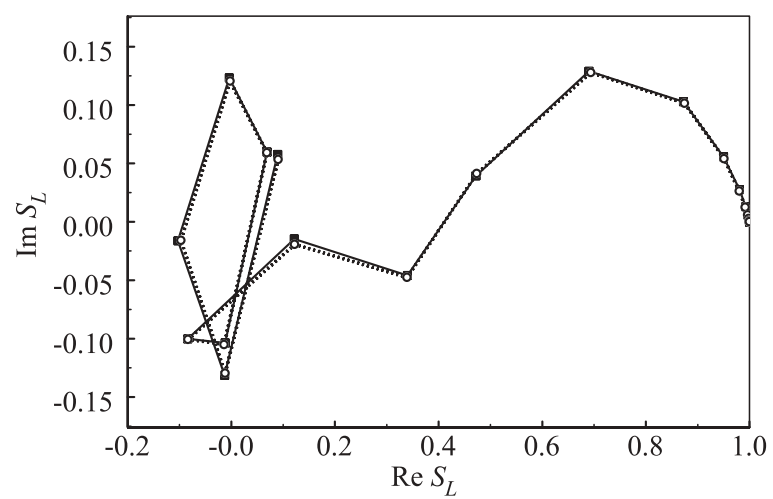

FIG. 5. Argand plot for the elastic $S$-matrix elements for the reaction $d+{ }^{58} \mathrm{Ni}$ at $E_{\mathrm{lab}}=21.6 \mathrm{MeV}$ calculated within the CDCC (open circles connected by dotted lines) and WPCD (black squares connected by solid lines) methods. 


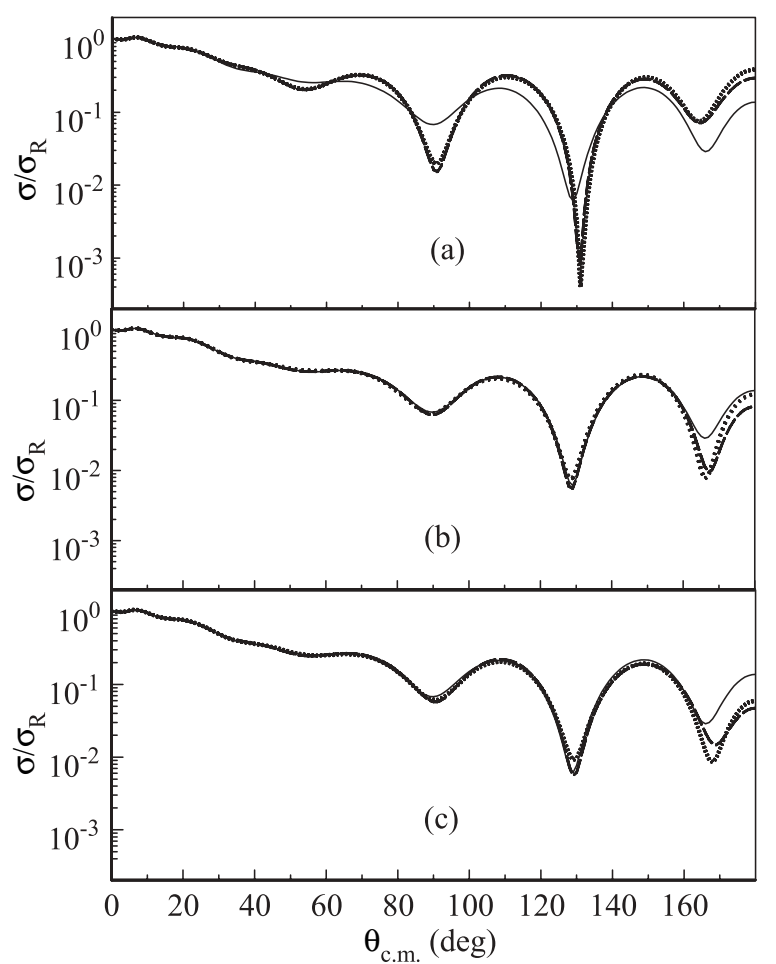

FIG. 6. Elastic $d+{ }^{58} \mathrm{Ni}$ cross section at $E_{\text {lab }}=12 \mathrm{MeV}$ calculated within the CDCC (dotted line) and WPCD (dashed line) approaches for different values of $E_{\max }$ : (a) $10.2 \mathrm{MeV}$, (b) $24.5 \mathrm{MeV}$, and (c) $36 \mathrm{MeV}$. The full curves correspond to the converged CDCC results $\left(E_{\max }=80 \mathrm{MeV}\right)$.

effect, which has not been seen in the two previous cases, begins to play some role at rather low incident energies.

\section{DISCUSSION}

The close agreement between the WPCD and R-matrix CDCC approaches found in the present work can be interpreted as a good indicator that the basic assumptions (or approximations) adopted in the WPCD method, in particular

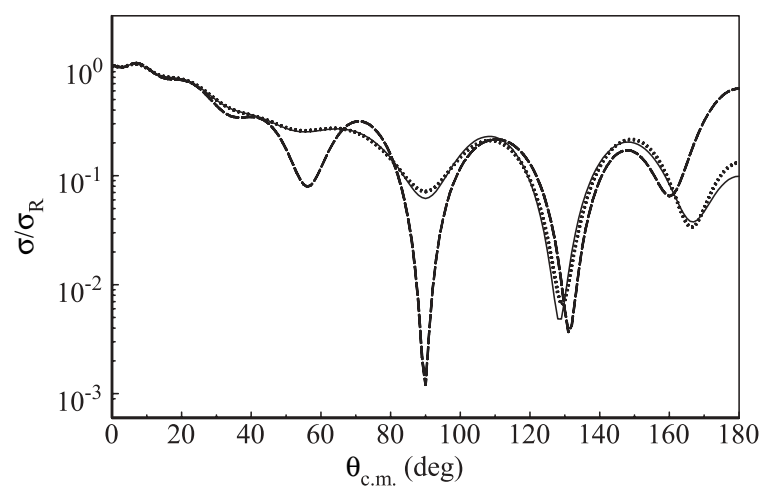

FIG. 7. Converged results (with respect to $E_{\max }$ ) for the differential elastic cross section (relative to Rutherford) for $d+{ }^{58} \mathrm{Ni}$ at $E_{\text {lab }}=12 \mathrm{MeV}$, calculated with the CDCC (dotted curve) and WPCD (solid curve) approaches. The dashed line corresponds to the folding calculation, in which continuum channels are not taken into account. the employment of orthonormalized stationary wave packets instead of exact nonnormalizable three-body scattering wave functions, are well justified and correct. This fact implies some further consequences in the whole many-body scattering theory. Actually, the usage of the total continuum discretization on the basis of normalizable (of $L^{2}$ type) stationary wave packets is equivalent to the formulation of the many-body scattering problem in a finite multidimensional box and leads a treatment of the original scattering problem analogous to the much more simple bound-state problem. In turn, the last conclusion allows us to treat the many-body scattering problem in similar way as the many-body bound states, e.g., by expanding the $L^{2}$-type stationary wave packets (constructed from the exact unknown scattering wave functions) on some convenient $L^{2}$ basis widely used in bound-state problems (for example, the many-body harmonic oscillator basis). The first results obtained by two of the present authors [21] with the application of this technique to the solution of the Faddeev equations above the three-body breakup threshold seem to support this expectation.

Another nice feature of the WPCD approach is the possibility to reformulate in a straightforward way the whole problem in terms of the Feshbach projection technique [23] and to include all the breakup channels into the respective Feshbach polarization nonlocal potential. After this, one can readily use the full applicability of the WPCD approach to the resulted nonlocal interactions (in contrast to the conventional coupled-channel method) and to reduce in a standard way the complicated many-body scattering problem to the simple matrix equations [27].

On the other hand, the very close agreement between the conventional CDCC approach and the fully alternative wavepacket integral method should remove any doubts reported in literature $[16,18]$ concerning the reliability and convergence properties of the Schrödinger coupled-channel scheme in the framework of the three-body scattering problem.

\section{CONCLUSION}

In this work, we have compared two essentially different approaches based on the general idea of continuum discretization for solving three-body scattering problems. The CDCC method was proposed to solve the coupled-channel problem in the framework of the Schrödinger equation formalism. This approach uses a discretization of the projectile internal continuum only, along with an explicit matching between inner and asymptotic scattering wave functions to extract the multichannel $S$ matrix. On the other hand, the WPCD approach is based on the total three-body continuum discretization which allows the use of the integral equation formalism of the scattering theory. The special wave-packet technique allows one to construct finite-dimensional analogs for all the scattering operators and to use only inner parts of wave functions in order to find the observables.

The direct comparison of both methods leads to the following conclusions:

(i) The two methods of continuum discretization discussed in this work produce very close results for the 
composite-particle elastic scattering at rather high and also at low energies.

(ii) Different types of discretization of the projectile internal sub-Hamiltonian continuum used in both methods do not lead to visible discrepancies at high and intermediate energies. Small differences in the elastic cross sections arise only at low incident energies. An employment of a highly nonuniform discretization distribution in the WPCD approach still leads to wellconverged results.

(iii) The effect of closed channels is not seen at high incident energies. This effect begins to play a visible role at intermediate energies (at the most backward angles) and cannot be ignored as it was assumed in some previous CDCC calculations. The convergence of the calculated cross section when increasing the number of included CCs depends only on the maximum excitation energy and is not sensitive to the details of the continuum discretization.

(iv) The effect of CC on elastic scattering becomes more significant when the incident energy of the projectile decreases. (v) The very close agreement between the results obtained with these two different approaches at various energies, from low to high, seems to indicate that the threebody wave-packet technique can be considered as a rather reliable and numerically accurate method for calculating composite-projectile scattering by nuclei. Simultaneously, one can confirm again that the conventional (or R-matrix) CDCC method leads to quite reliable and well-converged results for the elastic and inelastic scattering of composite projectiles.

\section{ACKNOWLEDGMENTS}

Authors V.I.K. and O.A.R. are thankful to Dr. V. N. Pomerantsev for numerous fruitful discussions. They also appreciate very much partial financial support from the RFBR Grant 07-02-00609, the joint RFBR-DFG Grant 0802-91959, and the President Grant MK-202.2008.2. A.M.M. acknowledges a research grant from the Junta de Andalucía and financial support from the DGICYT under Project FPA200613807-c02-01.
[1] R. C. Johnson and P. J. R. Soper, Phys. Rev. C 1, 976 (1970).

[2] G. H. Rawitscher, Phys. Rev. C 9, 2210 (1974).

[3] B. Anders and A. Lindner, Nucl. Phys. A296, 77 (1978).

[4] R. Y. Rasoanaivo and G. H. Rawitscher, Phys. Rev. C 39, 1709 (1989).

[5] J. P. Farrell, C. M. Vincent, and N. Austern, Ann. Phys. (NY) 96, 333 (1976); 114, 93 (1978).

[6] Z. C. Kuruoglu and F. S. Levin, Phys. Rev. Lett. 48, 899 (1982); R. Kozack and F. S. Levin, Phys. Rev. C 36, 883 (1987) .

[7] M. Yahiro, M. Nakano, Y. Iseri, and M. Kamimura, Prog. Theor. Phys. 67, 1467 (1982).

[8] M. Takashina, S. Takagi, Y. Sakuragi, and Y. Iseri, Phys. Rev. C 67, 037601 (2003); T. Egami, K. Ogata, T. Matsumoto, Y. Iseri, M. Kamimura, and M. Yahiro, ibid. 70, 047604 (2004).

[9] R. A. D. Piyadasa, M. Kawai, M. Kamimura, and M. Yahiro, Phys. Rev. C 60, 044611 (1999).

[10] N. C. Summers, F. M. Nunes, and I. J. Thompson, Phys. Rev. C 74, 014606 (2006).

[11] I. J. Thompson, Comput. Phys. Rep. 7, 167 (1988).

[12] P. Chau Huu-Tai, Nucl. Phys. A773, 56 (2006).

[13] A. M. Moro, J. M. Arias, J. Gomez-Camacho, I. Martel, F. Perez-Bernal, R. Crespo, and F. Nunes, Phys. Rev. C 65, 011602(R) (2001).

[14] A. M. Moro, F. Perez-Bernal, J. M. Arias, and J. GomezCamacho, Phys. Rev. C 73, 044612 (2006).
[15] A. M. Moro, F. M. Nunes, D. Escrig, and J. Gomez-Camacho, Nucl. Phys. A787, 463 (2007).

[16] T. Sawada and K. Thushima, Prog. Theor. Phys. 76, 440 (1986).

[17] N. Austern and M. Kawai, Prog. Theor. Phys. 80, 694 (1988).

[18] E. F. Redish, in Modern Three-Hadron Physics, edited by A. W. Thomas (Springer, Berlin, 1977), p. 181.

[19] V. I. Kukulin and O. A. Rubtsova, Theor. Math. Phys. 130, 54 (2002); 134, 404 (2003).

[20] V. I. Kukulin and O. A. Rubtsova, Theor. Math. Phys. 145, 1711 (2005).

[21] V. I. Kukulin, V. N. Pomerantsev, and O. A. Rubtsova, Theor. Math. Phys. 150, 403 (2007).

[22] V. I. Kukulin and O. A. Rubtsova, Phys. Rev. C 76, 047601 (2007).

[23] O. A. Rubtsova and V. I. Kukulin, Phys. At. Nucl. 70, 2025 (2007).

[24] A. Deltuva, A. M. Moro, E. Cravo, F. M. Nunes, and A. C. Fonseca, Phys. Rev. C 76, 064602 (2007).

[25] I. J. Thompson, in Scattering, edited by E. R. Pike and P. C. Sabatier (Academic, New York, 2001), p. 1360.

[26] A. J. Koning and J. P. Delaroche, Nucl. Phys. A713, 231 (2003).

[27] V. I. Kukulin, V. N. Pomerantsev, and O. A. Rubtsova, Nucl. Phys. A (to be published). 